\title{
HEARING RESULTS OF TYMPANOPLASTY WITH ATTIC RECONSTRUCTION VS. OBLITERATION IN ATTIC CHOLESTEATOMA
}

\author{
Slobodan Spremo ${ }^{1}$, Mirjana Gnjatic ${ }^{1}$, Sanja Spiric $^{1}$, Predrag Spiric ${ }^{1}$, Dmitar Travar ${ }^{1}$, \\ Mira Spremo ${ }^{2}$ \\ ${ }^{1}$ Department of Otorhinolaryngology, Univeristy Hospital Clinical Center, Banja Luka \\ ${ }^{2}$ Medical Faculty, Univeristy of Banja Luka
}

\begin{abstract}
INTRODUCTION: The aim of the study was to compare hearing results between tympanoplasty with attic wall reconstruction and attic obliteration.

MATERIAL AND METHODS: A prospective study included 88 patients who were operated for chronic suppurative otitis media (CSOM) and cholesteatoma in the ENT Department University Clinic Center Banja Luka from 2006 to 2011. Analysis of variance (ANOVA) was conducted to assess the impact of two different techniques of tympanoplasty: attic wall reconstruction and attic wall obliteration on patients' hearing. Hearing outcome was measured comparing preoperative to postoperative pure-tone average (PTA) and postoperative air-bone gap (ABG).

RESULTS: Cholesteatoma was found in $54(61.4 \%)$ patients: $31(35.2 \%)$ sinus cholesteatoma, $20(22.7 \%)$ attic cholesteatoma, 3 (3.4\%) tensa tympanic cholesteatoma, and 34 cases (38.6\%) of CSOM. Lateral attic wall and ossicular chain reconstruction was performed in 33 cases with subsequent attic reconstruction if the long process of the incus and the incudo-stapedial joint were missing. A mixed between-within-subjects analysis of variance showed significant improvement of PTA postoperatively in comparison to preoperative levels: Wilk's Lambda $=0.33, F(1,86)=172.1, p<0.001$. ANOVA showed significant improvement of ABG postoperatively in comparison to preoperative levels: Wilk's Lambda $=0.23, F(1,86)=286.3, p<0.001$. Hearing improvement was measured in both cases, the ABG closure and mean PTA improvement was larger in the attic reconstruction compared to attic obliteration technique.
\end{abstract}

CONCLUSION: Attic wall reconstruction with cartilage provides better postoperative hearing compared to attic obliteration. Attic reconstruction in revision cases for cholesteatoma recurrence is not recommended.

Keywords: tympanoplasty; cholesteatoma; hearing loss surgery

Address for correspondence:

Slobodan Spremo

Univeristy Hospital Clinical Center, Banja Luka

12 beba No.1

78000 Banja Luka, Bosna and Herzegovina

e-mail:spremosl@gmail.com

Received: August 4, 2015

Accepted: September 3, 2015

\section{INTRODUCTION}

Hearing improvement is a major factor in the assessment of the success of surgery for chronic otitis media. It could be achieved by meticulous reconstruction of the bony tympanic walls and ossicular chain at the same time (1). Attic cholesteatoma extends from the Prussack space in the lateral attic to the upper and medial. The common surgical approach to the attic is through lateral atticotomy by re- 
Slobodan Spremo, Mirjana Gnjatic, Sanja Spiric et al.

moving part of the superior and lateral bony wall of the external auditory meatus. This way malleus head, incus body and lateral attic space could be exposed $(2,3)$. Canal wall down tympanoplasty with subsequent posterior canal wall reconstruction or mastoid obliteration provides wide exposure of the tympanic and mastoid cavity and safe removal of the cholesteatoma extended in the mastoid anthrum and cells. The hearing results of different tympanoplasty techniques is affected by many factors: the extent of cholesteatoma or inflammatory granulations in the attic, destruction on the malleus and incus body and form of tympanoplasty performed (4). The main advantages of attic reconstruction are good aeration of the tympanic and mastoid cavity, well-preserved mucosa lining in the tympanum, large aerated space in the tympanum that closely resembles natural anatomic conditions in the middle ear. Adversely, approach to the posterior and medial attic space is limited, and most authors report higher residual and recurrent cholesteatoma rate compared to open tympanoplasty technique. Reduced volume of the tympanic cavity and widening the external auditory canal produces deteriorated anatomic condition and mostly unsatisfactory hearing results. The open tympanoplasty technique associated with attic reconstruction or obliteration is aimed to overcome major shortcomings of the posterior wall removal: having the large open postoperative cavity in the mastoid $(4,5)$ and susceptibility to periodic cavity infections. Lower frequency of postoperative ENT checkups and hearing improvement are also a benefit of attic reconstruction and obliteration technique $(5,6)$.

Postoperative hearing results are affected in many was by combination of the known preoperative and operative risk factors defined as Middle Ear Risk Index by Kartush and Black $(7,8)$. The risk factors: otorrhea, perforation size and location on the tympanic membrane, presence of middle ear granulations, cholesteatoma and the period of secretion were expressed as a numeric ratio. Few studies have evaluated hearing results of tympanoplasty with attic wall reconstruction versus obliteration.

We have evaluated the extent of the pathology related to chronic suppurative otitis media (CSOM) in the tympanic cavity, the condition of the osseous tympanic walls and condition of the ossicular chain in relation to preoperative and postoperative hearing.
The aim of this study was to estimate whether lateral attic wall reconstruction with autologous cartilage enables optimal anatomical and hearing results compared to canal wall down tympanoplasty with attic obliteration.

\section{MATERIAL AND METHODS}

In the prospective study we followed up patients who were operated for chronic otitis media in the Otorhinolaryngology department University Clinic Center Banja Luka from 2008 to 2014. We included 88 patients operated for attic cholesteatoma: the mean age was 46.1 years (range: $5-78$ years, $S D=16.1$ ). We have evaluated intraoperative records of the pathology on the tympanic bony walls, pathology on the ossicular chain and hearing preoperatively and postoperatively. The preoperative inclusion criteria were chronic suppurative otitis media (CSOM) with cholesteatoma originating in the attic. Postoperative follow up was taken 6 months following the operation. Extent of the cholesteatoma was assessed by a temporal bone CT scan.

\section{Surgical procedures}

The operation was performed under general anesthesia, by retroauricular approach. If the cholesteatoma matrix and granulation tissue could be safely removed from the lateral, upper attic and ossicles bodies we would perform just lateral atticotomy with subsequent attic reconstruction. If the ossicular chain was disrupted the osiculoplasty with autologous bone was performed. For cholesteatoma extended in the medial attic, retrotympanum or mastoid, we would disarticulate incudo-stapedial joint, remove incus and cut the head of the malleus. The cholesteatoma matrix could be traced then from the attic backwards if necessary into the anthrum by drilling the posterior and superior canal wall.

Cartilage graft were taken from concha and thinned into single $0.5 \mathrm{~mm}$ thick plate shaped to the bony defect. If both malleus and incus were missing, and the stapes was present we decided between following options:

1. In cases with intact external auditory canal wall:

a) grafting of the tympanic membrane with temporal muscle fascia that was placed on the capitulum of the stapes. 
b) Thin cartilage plate was placed on stapes capitulum to prevent postoperative tympanic retraction.

2. If the external auditory canal was taken down or was removed we used two procedures: attic wall reconstruction with cartilage or attic obliteration.

a) The head of the stapes was put in contact with the cartilage and free fascia graft. This form of type III tympanoplasty with attic reconstruction was mostly used to reconstruct for extensive cholesteatoma.

b) If malleus and incus were missing, we performed attic obliteration with bony chips taken from the mastoid cortex in combination with mastoid obliteration.

\section{Hearing assessment}

Pure tone audiometry was recorded preoperatively and 6 months postoperatively. For estimation of hearing we recorded Pure Tone Average (PTA) as a mean of hearing threshold on the $1000 \mathrm{~Hz}$, and Air Bone Gap (ABG) as the median on the $1000 \mathrm{~Hz}$ audiogram.

\section{Statistics}

Statics was calculated by the SPSS for Windows. For hearing levels we calculated mean, SD and variance for PTA and ABG. A mixed between-within Table 1. Correlation of cholesteatoma extension to attic obliteration vs. reconstruction

\begin{tabular}{lc|c|c|c|}
\multirow{2}{*}{ Cholesteatoma extension in the attic } & \multicolumn{2}{|c|}{ Operation } & Total \\
\cline { 3 - 5 } & & Attic Reconstruction & Attic Obliteration & 14 \\
Lateral attic cholesteatoma & $\mathrm{N}$ & 12 & 2 & $15,9 \%$ \\
& $\%$ & $30,0 \%$ & $4,2 \%$ & 20 \\
Lateral and medial attic & $\mathrm{N}$ & 9 & 11 & $22,7 \%$ \\
cholesteatoma & $\%$ & $22,5 \%$ & $22,9 \%$ & 54 \\
Attic, posterior tympanum, & $\mathrm{N}$ & 19 & 35 & $61,4 \%$ \\
mastoid & $\%$ & $47,5 \%$ & $72,9 \%$ & 88 \\
& $\mathrm{~N}$ & 40 & 48 & $100,0 \%$ \\
\hline \multirow{2}{*}{ Total } & $\%$ & $100,0 \%$ & $100,0 \%$ & \\
\hline \hline
\end{tabular}

Table 2. Correlation of ossicular chain reconstruction to attic obliteration vs. reconstruction

\begin{tabular}{|c|c|c|c|c|}
\hline \multirow{2}{*}{\multicolumn{2}{|c|}{ Ossicular chain reconstruction }} & \multicolumn{2}{|c|}{ Attic operation } & \multirow{2}{*}{ Total } \\
\hline & & Attic Reconstruction & Attic Obliteration & \\
\hline \multirow{2}{*}{ Ossicular chain mobilization } & $\mathrm{N}$ & 2 & 0 & 2 \\
\hline & $\%$ & $5,0 \%$ &, $0 \%$ & $2,3 \%$ \\
\hline \multirow{2}{*}{ Incus transposition } & $\mathrm{N}$ & 31 & 7 & 38 \\
\hline & $\%$ & $77,5 \%$ & $14,6 \%$ & $43,2 \%$ \\
\hline \multirow{2}{*}{ Collumela } & $\mathrm{N}$ & 6 & 19 & 25 \\
\hline & $\%$ & $15,0 \%$ & $39,6 \%$ & $28,4 \%$ \\
\hline \multirow{2}{*}{$\begin{array}{l}\text { Fascia or cartilage graft on } \\
\text { stapes }\end{array}$} & $\mathrm{N}$ & 0 & 7 & 7 \\
\hline & $\%$ &, $0 \%$ & $14,6 \%$ & $8,0 \%$ \\
\hline \multirow{2}{*}{ No reconstruction / neocavum } & $\mathrm{N}$ & 1 & 15 & 16 \\
\hline & $\%$ & $2,5 \%$ & $31,3 \%$ & $18,2 \%$ \\
\hline \multirow{2}{*}{ Total } & $\mathrm{N}$ & 40 & 48 & 88 \\
\hline & $\%$ & $100,0 \%$ & $100,0 \%$ & $100,0 \%$ \\
\hline
\end{tabular}


Slobodan Spremo, Mirjana Gnjatic, Sanja Spiric et al.

subjects analysis of variance (ANOVA) was conducted to assess the impact of attic wall reconstruction vs. attic wall obliteration on patients' hearing.

\section{RESULTS}

As shown in Table 1 , in most cases with limited lateral or upper attic cholesteatoma confinement we performed attic reconstruction with ossicular chain autologous bone reconstruction. Ways of ossicular chain reconstruction are shown in Table 2. Ossicular chain mobilization and incus transposition was achieved in 33 cases with subsequent attic reconstruction if the long process of the incus and the incudo-stapedial joint were missing. In these cases we opened the lateral and posterior attic and exposed the long process and part of the incus body, removed cholesteatoma sac and primarily reconstructed the lateral and posterior tympanic wall with cartilage.

Ossiculoplasty with autologous incus graft placed on stapes is shown on Figure 1, followed by reconstruction of the lateral attic wall with cartilage plate that was shaped after the bony defect in the attic on Figure 2. The cartilage was placed over the malleus head covering part of the long incus process and graft on the stapes capitulum. The cartilage graft and reconstructed ossicular chain is covered with free fascia graft. If the malleus and incus were missing we obliterated attic with autologous bone chips and bone pate up to the level of horizontal facial canal as shown on Figure 3.

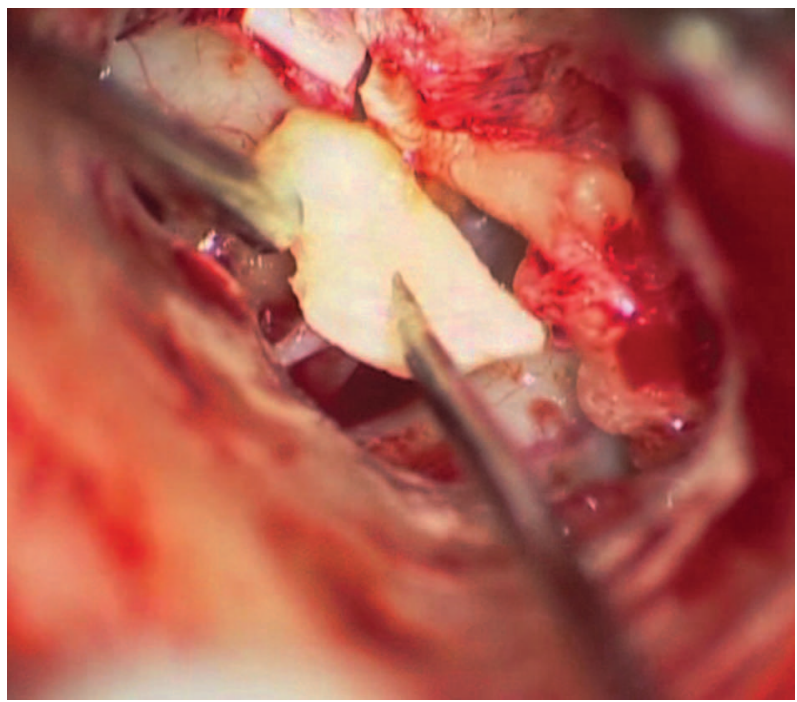

Figure 1. Ossiculoplasty, placin the incus graft on the ctapes

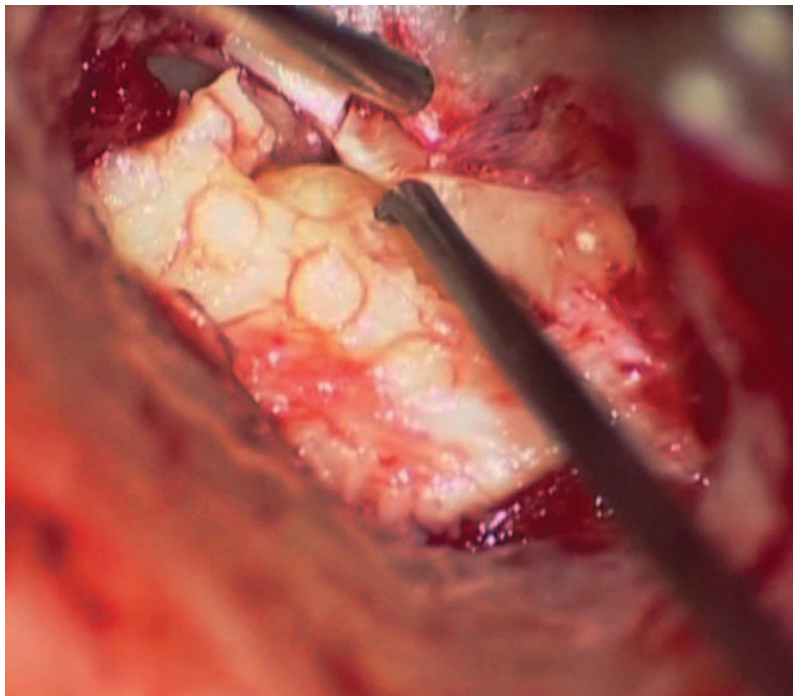

Figure 2. Reconstruction of the lateral attic wall with cartilage graft

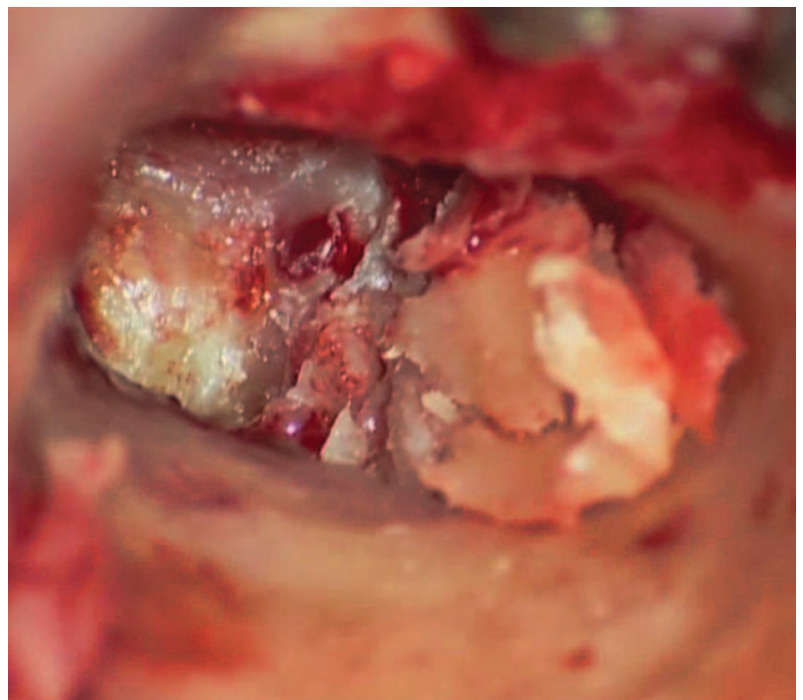

Figure 3. Obliteration of the attic with autologous bone chips and bone pate

Hearing results were evaluated preoperatively and 6 months postoperatively. Hearing improvement was better in attic reconstruction technique: mean hearing threshold (PTA) gain was $18.3 \mathrm{~dB}$ while in attic obliteration patients PTA was $10.7 \mathrm{~dB}$. The airbone gap closure as a parameter of hearing improvement was also greater in attic reconstruction compared to attic obliteration patients. Mean air-bone gap gain in the attic reconstruction group was 20.9 $\mathrm{dB}$, in comparison to $13.5 \mathrm{~dB}$ gain in attic obliteration patients. 
A mixed between-within-subjects analysis of variance (ANOVA) was conducted to assess the impact of the two different techniques of tympanoplasty: attic wall reconstruction and attic wall obliteration on patients' hearing following the operation. There was significant interaction between the dependent variables: preoperative-postoperative PTA, Wilk's Lambda $=0.91, \quad F(1,86)=9.1, \quad \mathrm{p}<0.001$, partial eta squared $=0.095$. We also observed significant interaction between the preoperative-postoperative $A B G$ and attic reconstruction vs. obliteration. Wilk's Lambda $=0.89, \mathrm{~F}(1,86)=10.7, \mathrm{p}<0.001$, partial eta squared $=0.011$. Statistic implies that larger the preoperative destruction on the attic bony wall was, the larger the preoperative hearing loss in term of pure-tone average and air-bone gap was in a reviewed population.

The statistic implies that both tympanoplasty techniques: attic reconstruction and attic obliteration achieved postoperative hearing improvement. The amount of hearing improvement measured by both, the ABG closure and mean PTA improvement was larger in the attic reconstruction compared to attic obliteration technique.

\section{DISCUSSION}

Pathology related to CSOM and cholesteatoma in the attic implies that through surgical approach it should be sufficient to expose the ossicles, associated pathology and enable the surgeon to remove it completely. Lateral atticotomy provides safe approach to the lateral and upper attic space and bodies of the malleus and incus. In cases when the ossicular chain is still intact, the cholesteatoma sac can be removed from the lateral attic and the attic wall is reconstructed with one piece of $0.5 \mathrm{~mm}$ thick autologous cartilage $(1,3,7)$. As anatomic conditions are restored almost completely, hearing results in terms of improving PTA and air-bone gap closure are the best. Bony erosion on the lateral attic wall and posterior external meatus wall point to severe cholesteatoma destruction in the attic on the ossicular chain and is highly suspected of extending into the mastoid anthrum on one side or the posterior tympanum along the long incus process. In our data cholesteatoma limited to the lateral attic space occurred rarely, in 14 cases (14.9\%). Destructions on the upper and posterior part of the external auditory canal along with re- traction in the posterior tympanic space were common found in $\mathrm{n} 54$ patients (61.4\%). In patients where cholesteatoma was limited to the lateral or upper attic, we performed attic reconstruction with ossicular chain autologous bone reconstruction. In these cases we opened the lateral and posterior attic and exposed the long process and part of the incus body, removed cholesteatoma sac and primarily reconstructed the lateral and posterior tympanic wall with cartilage. Ossicular chain mobilization and incus transposition was the best option with subsequent attic reconstruction if the long process of the incus and the incudo-stapedial joint were missing.

If the cholesteatoma extended to the medial attic, epitympanic recess, tympanic tubal orifice we usually disarticulated incudo-stapedial joint and removed incus entirely. Then the neck and head of the malleus was cut out and removed exposing the entire attic space. Usually that allowed for safe cholesteatoma removal from the attic space. We achieved the best hearing results if ossicular chain was reconstructed in two ways: If the stapes suprastructures were present, thin cartilage plate was placed over the capitulum and covered with temporalis fascia for closing the tympanic membrane perforation and reinforcing it from further retraction the same time. In cases where incus or stapes suprastructures were completely missing, osiculoplasty was performed by collumela which was made of cortical bone and shaped to the height of the outer part of hypotympanum. The collumela was placed directly on the stapes footplate. This is similar to the conclusion of the studies that observed the results of atticotomy to hearing and recurrence rate by Kim et al. (1) and Gerhrking (2).

Wide lateral atticotomy could be appropriate technique for approaching cholesteatoma extending under the head of the malleus into the mezotympanum or under and behind the long process of the incus into the oval and round window niche $(1,10)$. Incudo-stapedial joint disarticulation and removal of the incus provides proper operative space for removal of the pathology with proper anatomic landmarks in the tympanic cavity. If the cholesteatoma is limited to the tympanic cavity this procedure prevents for opening the mastoid air cells. Many authors favor canal wall up tympanoplasty (CWUp) with attic reconstruction over canal wall down tympanoplasty 
Slobodan Spremo, Mirjana Gnjatic, Sanja Spiric et al.

(CWD) $(2,5)$. Although the intraoperative visualization of the posterior tympanic space and mastoid in CWUp tympanoplasty is limited and cholesteatoma recurrence rate is higher, it provides close to anatomic shape of the postoperative tympanic cavity, proper basis for ossicular reconstruction and consequently better hearing then CWD tympanoplasty $(9,10)$. Due to large postoperative cavity in the attic, tympanic membrane retraction could occur. It is best prevented by reinforcing the lateral wall with cartilage strut. If we had preoperative evidence of severe tubal dysfunction we also reinforced the tympanic fascia graft with a thin piece of cartilage. Some authors $(1,4)$ report tympanic retraction in the attic as a main cause of tympanoplasty failure that occurs year following surgery. In our experience bone chips were prone to gradual resorption and further retraction of the tympanic membrane. Cartilage on the other hand is resistant to resorption and infection. We found it also a better hearing conductor on the tympanic membrane part as well.

Our results show that hearing improvement was greater in attic reconstruction technique: mean PTA gain preoperatively to postoperatively was 18.3 $\mathrm{dB}$ while in attic obliteration patients mean PTA gain was $10.7 \mathrm{~dB}$, and the hearing improved significantly $\mathrm{p}<0.001$. The air-bone gap closure was also greater in attic reconstruction group compared to the attic obliteration patients. Mean air-bone gap gain in attic reconstruction group was $20.9 \mathrm{~dB}$, in comparison to $13.5 \mathrm{~dB}$ gain in attic obliteration patients. We also observed significant interaction between the preoperative-postoperative air-bone gap and attic reconstruction vs. obliteration. $\mathrm{F}(1,86)=10.7, \mathrm{p}<0.001$. Analysis of variance showed significant improvement of air-bone gap parameter postoperatively in comparison to preoperative levels: $\mathrm{F}(1,86)=286.3$, $\mathrm{p}<0.001$. Statistic reveal that the larger the preoperative destruction on the attic bony wall was, the larger the preoperative hearing loss in term of pure-tone average and air-bone gap was in a reviewed population. Both tympanoplasty techniques: attic reconstruction and attic obliteration achieved postoperative hearing improvement. The amount of hearing improvement was measured in both, the ABG closure and mean PTA improvement was larger in the attic reconstruction compared to attic obliteration technique. Stankovic (10) reported long term hear- ing improvement following CWUp tympanoplasty for cholesteatoma in comparison to CDW and obliteration technique.

\section{CONCLUSION}

Lateral attic wall reconstruction with a cartilage plate provides better postoperative hearing. Several ossicular reconstruction methods are possible depending on the pathology found. For successful attic reconstruction some preconditions should be met: intact at least head of the malleus, sufficient tubal function, intact mucosal lining at least at the medial attic wall. We do not advocate attic reconstruction in revision cases for cholesteatoma recurrence.

\section{REFERENCES}

1. Kim JH, Choi SH, Chung JW. Clinical results of atticoantrotomy with attic reconstruction or attic obliteration for patients with an attic cholesteatoma. Clin Exp Otorhinolaryngol. 2009; 2(1): 39-43.

2. Gehrking E. Osteoplastic atticoantrotomy with autologous bone chips and a bony attic strut in cholesteatoma surgery. Eur Arch Otorhinolaryngol. 2010 Jul;267(7):1055-66.

3. Bacciu A, Pasanisi E, Vincenti V, Di Lella F, Bacciu S. Reconstruction of outer attic wall defects using bone paté: Long-term clinical and histological evaluation. Eur Arch Otorhinolaryngol. 2006 Nov;263(11):983-7.

4. Kim MB, Choi J, Lee JK, Park JY, Chu H, Cho YS, Hong SH, Chung WH. Hearing Outcomes According to the Types of Mastoidectomy: A Comparison between Canal Wall Up and Canal Wall Down Mastoidectomy. Clin Exp Otorhinolaryngol. 2010 Dec;3(4):203-6.

5. Kang MK, Ahn JK, Gu TW, Han CS. Epitympanoplasty with mastoid obliteration technique: a longterm study of results. Otolaryngol Head Neck Surg. 2009 May;140(5):687-91.

6. Dornhoffer JL, Gardner E. Prognostic factors in ossiculoplasty: a statistical staging system. Otol Neurotol. 2001 May;22(3):299-304.

7. Kartush JM. Ossicular chain reconstruction. Capitulum to malleus. Otolaryngol Clin North Am. 1994 Aug;27(4):689-715.

8. Black B. Ossiculoplasty prognosis: the spite method of assessment. Am J Otol. 1992 Nov;13(6):544-51.

9. Lee WS, Choi JY, Song MH, Son EJ, Jung SH, Kim $\mathrm{SH}$. Mastoid and epitympanic obliteration in canal 
Hearing results of tympanoplasty with attic reconstruction vs. obliteration in attic cholesteatoma

wall up mastoidectomy for prevention of retraction pocket. Otol Neurotol. 2005 Nov;26(6):1107-11.

10. Stankovic M. Follow-up of cholesteatoma surgery: open versus closed tympanoplasty. ORL J Otorhinolaryngol Relat Spec. 2007;69(5):299-305 